and the contents examined was a distinct advantage. By Braun's method, glass capillaries were the only ones possible but this improvement permits the use of any number of insoluble substances for a membrane.

2. In addition to the results obtained by Braun, electrostenolytic deposits were secured, by the new method, in water solutions of cuprous bromide, cuprous iodide, copper sulfate, Fehling's solution, sodium plumbite, sodium stannite, ammonium molybdate, manganese chloride and nickelous bromide. These results were secured with direct current at I Io v., but a deposit was also secured in a cobaltous bromide solution using alternating current.

A discharge of bromide ion was observed around an initial deposit of cobalt peroxide formed by electrostenolysis.

Earlham Colgege, Richmond, Indana.

\title{
THE ELECTRON CONCEPTION OF VALENCE.
}

By STUaRT J. Bates.

Received February 27, 1914.

There is one point of considerable importance in connection with this theory to which it appears desirable to call attention in view of the recent discussions of this topic in ThIS Journal. Comparatively recent works in physics has caused physicists to reconsider the validity of the assumption that "every chemical bond formed between two atoms involves the transfer of a corpuscle from one atom to the other." 1 Thus Sir J. J. Thomson, who proposed and first developed the electron theory of valence, has, chiefly as a result of his investigation of positive rays, changed his opinion regarding the mechanism of the attraction between atoms. "We are led by these results to regard the electrical forces which keep the atoms in a molecule together as due not to one atom being charged positively and the other negatively, but to the displacement of the positive and negative electricity in each atom. Thus each atom acts like an electrical doublet, and attracts another atom in much the same way that two magnets attract each other."2

The objections which may be raised against the electron theory of valence are probably much less widely known among chemists than is the evidence in favor of it. It seems worth while to indicate the nature of a few of these objections without attempting to develop them here:

(I) It is difficult to conceive of the two atoms in the molecule of an elementary gas as hydrogen, or of the two methyl groups in ethane, as being so different from one another as would be the case if one half of the mole-

1 Nelson, Beans and Falk, This JournaI, 35, 18 10 (1913).

2 J. J. Thomson, "Rays of Positive Electricity" p. 66, (1913), Longmans, Green $\&$ Co. See also Phil. Mag., 24, 249 (Igr2). 
cule is positive and the other negative. In the vast majority of reactions the two halves of such molecules react in an identical manner. ${ }^{1}$

(2) The electron theory of valence predicts a much greater number of isomeric compounds, even among relatively simple organic substances, than have as yet been discovered.

(3) According to this theory the greatest number of electrons which an atom can readily lose is the maximum positive valence number of the element. The method of positive rays indicate that there is no relation between these numbers. "The maximum number of charges carried by a multiply charged atom does not seem to be related to any chemical property of the atom such as its valency, but to depend mainly on the atomic weight." 2 The electron theory of valence indicates that the inert gases should give up electrons with great difficulty if at all. Helium readily loses two electrons, while the, argon atom may carry one, two or three positive charges and that of krypton four or five. Positively and negatively charged atoms of hydrogen, oxygen, sulfur and chlorine have been found. Nitrogen and mercury atoms appear to be capable of acquiring a positive charge only, ${ }^{3}$ the latter may lose any number from one to eight of electrons. Gases such as methane or carbon dioxide would be expected to yield carbon atoms carrying four positive or negative charges. Two appears to be the greatest number of charges which this atom is capable of acquiring. ${ }^{4}$ According to the electron theory of valence, the carbon atom in carbon monoxide is positively charged, while the oxygen atom is negatively charged. The method of positive rays shows that when a molecule of this gas is dissociated in the vacuum tube the numbers of positively charged oxygen atoms and of positively charged carbon atoms are practically equal. ${ }^{5}$

The spectral method of analysis has yielded evidence regarding the existence of charged atoms, substantiating that obtained by the electromagnetic method. ${ }^{6}$

(4) In an equilibrium mixture of a gas and its dissociation products, new molecules of the gas are being continually formed while others are breaking up. The electron theory of valence predicts that such a mixture would contain more charged atoms or molecules than do stable gases at the same temperature and pressure. Conductivity measurements have failed to indicate any difference in the number of charged particles in these two classes of gases. ${ }^{7}$

1 Thomson, "The Corpuscular Theory of Matter," p. ז30, et. seq. (1907).

${ }^{2}$ Idem., "Rays of Positive Electricity," p. 53.

"Idem., Proc. Roy. Soc., (A) 89, ro (I913).

4 Phil. Mag., 21, 239 (I911).

${ }^{5}$ Ibid., 24, 249 (1912).

${ }^{6}$ Stark, Physik, Z., I4, 961 (1913).

7 Phil. Mag., 24, 250 (I912). 
(5) One of the conclusions at which Kirkby arrived as a result of an investigation of the "Chemical Action of Electrical Discharge in Electrolytic Gas," was stated as follows: "The atoms of oxygen, when separated, are not charged electrically. This is proved by experiments independently of theory. Hence the atom of oxygen is not bound in a molecule of water vapor by electrostatic force." 1 Subsequent experiments by Kirkby and $\mathrm{Marsh}^{2}$ on the explosion of azoimide between charged plates leads to a similar conclusion.

(6) In order for the atoms in a molecule to be held together by electrical forces it is not necessary that it be assumed that an electron has moved from one atom to another. Thomson has considered a distribution of electrons within the outer sphere of the atom which accounts for two neutral atoms being held together. ${ }^{3}$ Stark, without employing the assumption that a chemical bond involves the transfer of an electron from one atom to another, has developed ${ }^{4}$ a system by which the atoms of a molecule may be held together by means of electrical forces. Bohr, ${ }^{5}$ assuming the structure which Rutherford ${ }^{6}$ has proposed for the atom, showed that two atoms may be attracted to one another by electrical forces even though both are electrically neutral as a whole. ${ }^{7}$ Such an electrical attraction between atoms involving no movement of an electron from one atom to another supports the idea of non-polar valences developed by Bray and Branch ${ }^{8}$ and Lewis. ${ }^{9}$ However, these authors consider polar valences also. Some of the difficulties which this dualistic view of valence presents have been pointed out by Falk and Nelson ${ }^{10}$ and by W. A. Noyes. ${ }^{11}$

It will thus be seen that on the whole the phenomena of physics are opposed to the view that in the molecule the atoms are charged with respect to one another, and to the theory of valence developed on this assumption. Chemistry contributes the most satisfactory evi-

${ }^{1}$ Proc. Roy. Soc., (A) 85, I5 I (IgII).

${ }^{2}$ Ibid., 88, 90 (Igr3).

${ }^{3}$ Engineering, 95, 329 (1913).

${ }^{4}$ Jahrb. Rad. Elektr., 9, 15 (1912); Z. Electrochem,, 19, 862 (1913); Prinzipien der Atomdynamik (I 1 10), Hirzel, Leipzig.

${ }^{5}$ Phil. Mag., 26, 857 (1913).

${ }^{6} \mathrm{Ibid}$., 2I, 669 (1911).

"Recently, additional support has been lent to this "non-polar" view of the mechanism of the combination of atoms to form molecules. In November, 1913, when Bohr calculated on the above assumptions that the heat of combination of hydrogen atoms to form hydrogen molecules was 60,000 calories per gram molecule, the accepted experimental value was 130,000 calories. Since then Langmuir (Phil. Mag., 27, 188 ( $\mathrm{x} 9 \mathrm{14})$ ) has published the results of further experiments which give the value 76,000 calories.

${ }^{8}$ This Journal, 35, I440 (I9I3).

'Ibid., 35, 1448 (I913).

30 Ibid., 36, 209 (1914).

11 Ibid., 36, 2 14 (I914). 
dence in its favor. It explains in a simple and clear manner certain reactions, particularly the oxidation and reduction of electrolytes in solution. This is sufficient justification for its application to such reactions. The fact that electrolytes dissociate in solution into oppositely charged ions is not, however, conclusive evidence that in the molecule the atoms or groups of atoms are electrically charged with respect to one another. Ionization may consist essentially in the passage of an electron from one atom or group to another, these then separate from one another forming the ions. The ease with which this movement of the electron takes place depends upon the nature both of the substance and of the solvent. The distinction, which is drawn between electrolytes and non-electrolytes is largely based upon the extent to which the substance is influenced by its environment. In the pure state as solid, liquid or gas, the molecule of an electrolyte differs but little in its behavior from that of other substances.

Nelson, Beans and Falk have given a general classification of reactions on the basis of the electron theory of valence. "In every reaction; I, If the algebraic sum of the positive and negative charges on a definite atom of the molecule changes; either $A$, the number of corpuscles on the atom increases; or $B$, the number of corpuscles on the atom decreases; II, If the algebraic sum of the positive and negative charges on the atom remains constant; either $A$, the arithmetrical sum changes; or $B$, the arithmetrical sum remains constant. Applied to chemical reactions, IA includes reduction reactions, IB adduction reactions, IIA onium compound formation, IIB metatheses in which none of the changes IA, IB or IIA takes place. Stated slightly differently, the classification includes reactions involving reduction, oxidation, onium compound formation, and simple replacement or rearrangement."' These authors have discussed these types of reactions and have explained the electronic changes which are involved in each of these various classes.

It would apparently be impossible to discover or even imagine any reaction which could not be classified and explained on such a basis. Thus, suppose some entirely new type of reaction were discovered. Then, on the basis of this theory, if the algebraic sum of the positive and negative charges on a definite atom changes during the reaction, by definition the reaction is one of oxidation-reduction and as such is explainable. If, on the other hand, the algebraic sum of the charges on a definite atom remain the same, the reaction is by definition an "onium" compound formation or a metatheses, which also may be explained by the electron theory of valence. From the nature of the definitions and the breadth of the classification, the discussion has been removed from dependence upon experimental data. In other words, a discussion on the basis of such a classification of the elec-

1 This Journai, 35, I8I5 (I9I3). 
tronic changes taking place during reactions can neither support or refute the theory. On the other hand, if the assumptions of the theory be granted, a useful method of classifying reactions may be derived.

A similar remark applies to the application of the theory to the structure of molecules. The electron theory has been applied by various authors to the structure of such chemical substances as chlorine, hydrogen peroxide, ammonium chloride, etc. If molecules such as these can be satisfactorily explained, it would seem that every conceivable molecule could be explained on the same basis. Apparently some new line of attack, such as that recently undertaken by $W$. A. Noyes, ${ }^{1}$ must be looked to for any decisive argument of a chemical nature for or against the theory.

The classification and relation of compounds and reactions, which has resulted from the application of the electron theory of valence, has been of value. The part played by its fundamental assumption has been largely a means of relating phenomena and the actual movement of the electron is not necessarily involved in the conclusion. That is, it is possible that some other assumption involving the ideas lately proposed by Thomson or those developed by Stark or by Bohr would lead to a similar classification of compounds and reactions.

UNIVERSITY OF ILLINOIS,

URBANA, ILL.

[CONTRIBUtion from the DIviston OF Physical Chemistry of the University of MINNESOTA.]

\section{AN APPARATUS FOR THE MEASUREMENT OF VAPOR PRES- SURES BY THE DYNAMIC METHOD AND DETER- MINATIONS OF THE VAPOR PRESSURE OF WATER AT $24.97^{\circ} .^{2}$}

By I. H. Derby, F. Daniels and F. C. Gutsche.

Received March 19, 1914.

It has been generally conceded that the dynamic, or "air bubbling," methods for determining vapor pressures of liquids offer considerable promise, but, owing to the more or less cumbersome and complicated apparatus required by such processes, these methods have not been utilized save in a few instances. Regnault ${ }^{3}$ in 1845 was seemingly the first to use the dynamic method. His procedure consisted in passing a known volume of air over a moist sponge and through moist silk screens, and weighing the water vaporized, thus obtaining values for the vapor pressure of water which agreed very closely with results obtained by static methods. This method of Regnault's was subsequently used with various modifica-

1 This Journal, 35,767 (1913).

2 Presented at the annual meeting of the American Chemical Society, December, IgIO.

${ }^{3}$ Ann. chim. phys., 3, 15, $129(1845)$. 